\title{
Siete mitos de la investigación aplicada y el desarrollo tecnológico universitario
}

\section{Seven Myths of the University Applied Research and Technology Development}

\author{
Vega-González L.R. \\ Coordinación de Vinculación y Gestión Tecnológica \\ Centro de Ciencias Aplicadas y Desarrollo Tecnológico, CCADET-UNAM \\ E-mail:Irvg@servidor.unam.mx; roberto.vega@ccadet.unam.mx
}

Información del artículo: recibido: enero de 2009, aceptado: abril de 2010

\section{Resumen}

A partir de diversas experiencias en proyectos de desarrollo tecnológico realizados en el Centro de Ciencias Aplicadas y Desarrollo Tecnológico, CCADET que es una de las entidades parte del subsistema de investigación científica de la Universidad Nacional Autónoma de México, UNAM, en este artículo se presentan una serie de mitos que se dan en el medio académico sobre la investigación aplicada y el desarrollo tecnológico (IA\&DT). La metodología, parte de la presentación de las experiencias empíricas para describir diferentes situaciones alrededor de los mitos. Estas situaciones se analizan posteriormente, con base en diferentes enfoques de gestión tecnológica que se presentan en la literatura actual. El contraste permite establecer reflexiones, comentarios y recomendaciones para cada uno de los mitos, los cuales son discutidos e integrados en la conclusión final. En la discusión se infiere que si en las universidades públicas mexicanas y tal vez inclusive en las Latinoamericanas, aprender a desarrollar tecnología es difícil, lo es más aún enfocarse a la tecnología correcta y comercializarla. Los procesos requeridos para realizar IA\&DT en el medio académico, dan origen a una serie de mitos y supuestos muy relacionados con nuestro contexto cultural, que hay que desmitificar.

\footnotetext{
Abstract

From a series of technology development project experiences at the Centro de Ciencias Aplicadas y Desarrollo Tecnológico, CCADET, part of the research subsystem of the Universidad Nacional Autónoma de México, UNAM, in this paper a series of myths circulating among the academic people about technology development and applied research $(R \mathcal{E} D)$ are presented. The methodology begins with the presentation of the empiric experiences to describe different myth situations. These situations are later analyzed based on different focuses existing in technology the management
}

\section{Descriptores}

- desarrollo tecnológico universitario en México

- gestión tecnológica

\section{Descriptores}

- third World University's technology development

- technology management 
present literature. This contrast permits to establish a partial conclusion for each one of the myths, all of them integrated at the end of the document. In the discussion, it is concluded that if in the public Mexican universities case and possibly in the Latin-American case, learning to develop technology is difficult; it is more, to focus in the right technology and commercialize it. The processes required for $R E D$ in the academic media, give birth to myths related with our cultural context that it is required to demystify.

\section{Introducción}

En una junta de aclaraciones para el desarrollo de un proyecto denominado "urna electrónica para el Instituto Electoral del Distrito Federal", el finado Gian Franco Bissiachi $^{1}$ (§), entonces representante del Centro de Tecnología de la Facultad de Estudios Profesionales de Aragón de la UNAM, manifestando su interés en participar en el desarrollo del proyecto y aceptando las retadoras condiciones ${ }^{2}$ de un presupuesto muy limitado, tiempos de entrega mínimos y alcances no tanto; externó: “... yo sí le entro, la tecnología se hace en el camino...", ya que en su experiencia, enfrentarse a los problemas era la mejor forma de aprender a hacer tecnología (learning by doing).

Tal y como estaba establecido en las bases del concurso, la institución patrocinadora asignó el proyecto a varios grupos de desarrollo de diferentes instituciones de enseñanza e investigación. Los ganadores fueron el Instituto Politécnico Nacional, la ENEP Aragón de la UNAM y el Tecnológico de Monterrey. Posteriormente se reportó en algunos medios de difusión que todas las instituciones terminaron sus prototipos exitosamente en tiempo y forma, y que el patrocinador evaluaría y decidiría entre los desarrollos entregados, aquel que mejor cumpliera con sus objetivos.

Como la anterior, existen muchas historias alrededor del desarrollo tecnológico universitario a partir de las cuales se genera toda una cultura de dichos, formas de pensar y pre conceptos entre los académicos relacionados con la IA\&DT. Dependiendo del contexto, algunos de ellos son verídicos y positivos, mientras que otros no lo son tanto. Las complejidades de la IA\&DT y de estas ideas dan origen a una serie de mitos o malos entendidos que complican la comunicación entre los distintos agentes involucrados y su entendimiento al interior y al exterior de la universidad. A continuación,

\footnotetext{
1 Investigador de la UNAM que participó en el Programa Universitario de Investigación Espacial (PUIDE) en la década de los años noventa del siglo pasado.

2 Condiciones por cierto muy comunes que son requeridas por los patrocinadores tanto públicos como privados para el financiamiento de este tipo de proyectos.
}

se presentan en forma sintética situaciones en las que se dan algunos de los mitos más comunes que hemos encontrado.

\section{Primer mito}

Cuando alguna organización externa a la universidad requiere el desarrollo de un paquete tecnológico y se pone en contacto con una facultad, con un instituto o con un centro universitario, la mayor parte de los académicos, investigadores, técnicos, ingenieros de desarrollo de la entidad universitaria siempre estarán de acuerdo y disponibles para participar en los proyectos de IA\&DT patrocinados.

\section{Situación alrededor del mito}

Aunque en una institución de IA\&DT exista un gran número de académicos, ya sea investigadores, técnicos académicos, ingenieros, diseñadores y personal de apoyo, esto no quiere decir que todos ellos se encuentran disponibles y receptivos para participar en proyectos de investigación aplicada o desarrollo tecnológico patrocinados por alguna organización externa. Lo que sí es seguro, es que prácticamente todos ellos estarán dispuestos a desarrollar prototipos académicos para investigación, experimentación o enseñanza, ya que hasta ahora, estos resultados permiten cumplir con los objetivos fundamentales de la UNAM. La situación es reforzada por el hecho de que a través de los resultados que así se obtienen se evalúa la actividad anual de los académicos por los consejos técnicos o académicos y esto influye en su contratación, en su promoción, o bien, en el nivel de estímulos económicos que el académico recibirá. Además, en proyectos académicos los tiempos de respuesta y en algunos casos inclusive los resultados, están dentro del control de los académicos, ya que generalmente se trata de proyectos con financiamiento interno; mientras que en el caso de proyectos contratados con la industria la exigencia y la responsabilidad es mucho mayor; es decir, hay que cumplir con tiempos de entrega más estrictos y los entregables deben satisfacer los requerimientos del patrocinador. Todo esto con el agravante de que, tal vez por los términos de confidencialidad del convenio, no esté permitido publicar 
los resultados obtenidos durante el proyecto. En suma, muy pocos académicos de una organización universitaria estarán dispuestos a participar en proyectos de IA\&DT por vocación, ya que en muchos casos las exigencias de los proyectos restan tiempo a los mismos para realizar otros proyectos internos, cuyos resultados probablemente puedan publicar.

Por su parte, los directivos de los centros e institutos con baja innovatividad no pueden forzar a que sus académicos participen en la realización de proyectos de IA\&DT, ya que por su naturaleza jurídica en dichas instituciones existe libertad de cátedra e investigación.

Análisis bajo el enfoque de gestión tecnológica

La actitud innovadora es un fenómeno que ha sido estudiado internacionalmente con enfoques de psicología del trabajo, antropología social y gestión tecnológica, técnica, artística, organizacional y universitaria, entre otros. En algunos países como Venezuela, se promueven eventos de gran escala buscando promover el desarrollo de la actitud innovadora y del emprendimiento. (Premio Eureka, 2008). En una organización productiva el término innovatividad se refiere a la actitud de los miembros de la organización ante proyectos innovadores, tales como la instalación de nueva maquinaria, de equipos e instrumentos modernos y eficientes, o bien, la implantación y uso de nuevos sistemas de software para la producción. Es un término relativo que intenta cuantificar como es que la gente reacciona a las nuevas cosas a partir de sus diferencias interindividuales (Godsmith et al., 2003). Por extensión, la innovatividad en una organización que desarrolla tecnología, también puede verse como la actitud de los académicos a participar en proyectos de IA\&DT.

Los estudios de innovatividad se han enfocado en tres contextos: de comportamiento, global y de personalidad. Dependiendo de la cultura organizacional los grupos académicos de centros o institutos de IA\&DT son propensos o adversos a la innovación. Si bien es cierto que en algunas instituciones los académicos no quieren dedicarse a desarrollar tecnología, debido a los esquemas de evaluación que imperan en sus entidades, en otras instituciones tales como el Centro de Tecnología Avanzada (CIATEQ, 2008) y el Centro de Investigación y Asistencia en Tecnología y Diseño del Estado de Jalisco, A.C, (CIATEJ, 2008) ${ }^{3}$, por citar solo algunos ejemplos, ocurre lo contrario. La misión de estas instituciones gira principalmente alrededor del desarrollo

\footnotetext{
3 Ambos parte del sistema de Tecnológicos dependientes de la Secretaría de Educación Pública (SEP) y del Consejo Nacional de Ciencia y Tecnología (CONACYT), en México.
}

tecnológico y por lo tanto, los esquemas de evaluación de su personal científico y tecnológico consideran sustancialmente los resultados que sus miembros obtienen en proyectos de desarrollo y de servicios tecnológicos. En la UNAM, algunas entidades miembros del Subsistema de la Investigación Científica como el Instituto de Ingeniería (II), el Instituto en Matemáticas Aplicadas y Sistemas (IIMAS), el Centro de Física Aplicada y Tecnología Avanzada (CFATA) y el Centro de Ciencias Aplicadas y Desarrollo Tecnológico (CCADET), entre otros, son ejemplos de las entidades universitarias en las que se están realizando esfuerzos para considerar los proyectos de desarrollo de tecnología exitosos en las evaluaciones de su personal académico.

\section{Reflexión, comentarios y recomendaciones}

La innovatividad de los grupos académicos que realizan desarrollo tecnológico depende fuertemente de la vocación de los académicos y de los objetivos y procedimientos de evaluación académica organizacionales. Si se quiere promover una actitud positiva entre los académicos dirigida a fomentar el desarrollo de proyectos de IA\&DT, parece ser que un par de requisitos fundamentales serán, por una parte, promover el desarrollo de proyectos que respondan a la solución de los grandes problemas que aquejan a la sociedad mexicana, tales como la educación, la salud, el desarrollo de tecnologías competitivas para las pequeñas empresas, la disminución de impactos ambientales, la disponibilidad de fuentes de energía alternas, el uso adecuado de los recursos como el agua, entre muchos otros, y por la otra, valorar adecuadamente la participación de los académicos en los mismos.

También es imperativo que al formar investigadores en los estudios de posgrado que se imparten en la UNAM, los profesores promuevan el desarrollo de la conciencia en los estudiantes sobre la importancia de que tengan actitud positiva y también participen en proyectos de IA\&DT para el bienestar del país a su vez contribuyendo a que la universidad cumpla con una de sus funciones primordiales.

\section{Segundo mito}

Los desarrollos tecnológicos son de bajo costo.

Situación alrededor del mito

Con frecuencia se presenta la situación de que algunos representantes de la industria o de diferentes organizaciones públicas o privadas visitan nuestros institutos o centros de IA\&DT y se organizan visitas guiadas en las que los investigadores o los académicos responsables 
presentan sus laboratorios o grupos académicos y algunas veces incluyen dispositivos tecnológicos tales como aparatos electrónicos, mecatrónicos o programas de software. Cuando los académicos dan su presentación o muestran sus resultados frecuentemente usan expresiones como: “...este dispositivo es de bajo costo, como usted puede ver para replicarlo sólo se requieren algunos materiales, una tarjeta electrónica y unos cuantos tornillos....". Por supuesto que estos comentarios son bienvenidos por los posibles patrocinadores de nuevos proyectos, pero son perjudiciales para cualquier negociación posterior, ya que fijan la posición mental del cliente, lo que podría conducir a uno de los tres casos siguientes:

a) En caso de que los clientes se interesaran por el desarrollo en cuestión, nunca aceptarán costos mayores de los de fabricación, porque "...el dispositivo ya está hecho, cuando mucho requiere pequeñas modificaciones...",

b) en caso de que los clientes se interesen por un nuevo desarrollo jamás aceptarán un presupuesto que vaya más allá de un valor mínimo de la tecnología, que formularán mentalmente, generalmente relacionado con los costos parciales de fabricación de los prototipos,

c) la imagen del grupo académico y, lo que es peor aún, de la institución en la mente de los clientes es que se trata de una institución "baratera".

Análisis bajo el enfoque de gestión tecnológica

Cuando se realiza la valoración adecuada de los productos de IA\&DT, normalmente encontraremos que su valor es más elevado de lo que se piensa. El valor de la tecnología no sólo debe incluir el costo de los materiales de construcción, sino que debe incluir el valor de los activos tangibles e intangibles que se generan a lo largo del desarrollo de la tecnología.

En la figura 1 podemos ver que el desarrollo de tecnologías se da en varias etapas. El principio de todo el proceso pueden ser las ideas que surgen de la investigación básica y que tratan de dar respuesta a alguna demanda detectada en el mercado. La primera etapa es la prueba de concepto (0-1), que inicia con la construcción de un dispositivo preliminar o prototipo de concepto con el cual se realiza la validación de la factibilidad técnica $\mathrm{y}$ funcional del dispositivo.

El prototipo de concepto consume recursos en la forma de materiales, equipo de prueba y de medición, salarios del personal técnico, administración y un porcentaje de indirectos asociado al proyecto. En este punto las tarjetas electrónicas, los ensambles mecánicos, ópticos, acústicos y de sensores se soportan en cajas o cubiertas improvisadas y se interconectan con montones de alambres. Las entradas y salidas no tienen terminales atornilladas, sino que se conectan con alambres y pinzas o "caimanes". Los despliegues son tomados en préstamo de otros experimentos y el sistema en su conjunto no tiene una gran exactitud ni precisión. En resumen, casi siempre los prototipos de concepto son artesanales. Los programas de software en su versión alfa $(\alpha)$ son considerados prototipos de concepto.

Entre los puntos 1 y 2 de la gráfica de la figura 1 se realizan intensas actividades de mejora que consumen una gran cantidad de recursos. Los grupos científicos ofrecen diferentes montajes experimentales de labora-

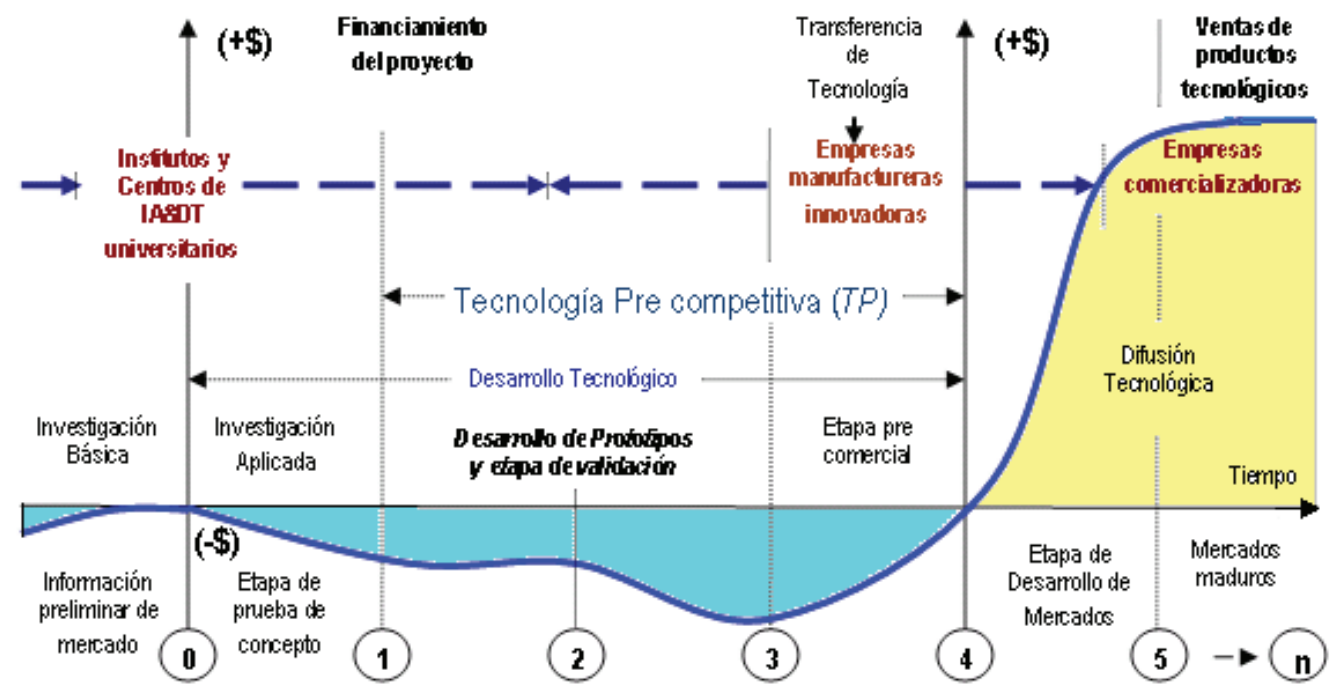

Figura 1. Etapas en la construcción de una tecnología precompetitiva 
torio y el personal técnico trabaja intensamente para obtener una versión mejorada del prototipo de concepto preliminar. Para cada mejora se requiere mucha iteración en los prototipos de desarrollo. Actividades como el ensamble, calibración, pruebas, diseños de ingeniería y modificaciones se realizan varias veces antes de terminar una nueva versión del prototipo. Este proceso puede tomar semanas, meses y hasta años dependiendo de la complejidad del proyecto y de la disponibilidad de materiales y recursos.

Al final de la etapa 2 el grupo de proyecto tiene un prototipo de banco (bench prototype) el cual es mejor que el prototipo de concepto, pero aún es considerado como una tecnología precompetitiva de bajo nivel. El prototipo de banco muchas veces incluye modificaciones mecánicas, eléctricas, ópticas, acústicas y de los ensambles de sensores. También se mejoran las tarjetas electrónicas y las carcasas o cajas de montaje. En esta etapa se usa equipo de calibración y pruebas de alta precisión y exactitud. Los programas de software en versión $\beta$ son considerados prototipos de banco. Entre las etapas 2 y 3 el equipo de proyecto debe trabajar en nuevas características y especificaciones, además el proyecto se extiende hacia los grupos de ingeniería y diseño, los cuales deben trabajar en aspectos como la usabilidad, la confiabilidad y la disponibilidad del dispositivo tecnológico. También se trabaja en el aspecto exterior mejorando los contenedores y los despliegues. Al final de la etapa 3 se obtiene un prototipo de laboratorio que ya tiene el carácter de tecnología precompetitiva total. Casi siempre es el fin de la intervención de los grupos académicos universitarios que realizan IA\&DT en los proyectos de innovación. En este momento se procede a obtener los certificados o títulos de propiedad intelectual que puedan resultar del prototipo tecnológico obtenido en el proyecto de desarrollo. Cuando los prototipos fueron desarrollados con financiamiento externo, el siguiente paso son los trabajos de transferencia de tecnología de los derechos de propiedad intelectual y la negociación de sus términos. Generalmente se requiere una corrida de valuación tecnológica para definir los términos de los contratos de TT. Una vez que se realiza la transacción, la tecnología pasa a ser un activo tangible. La TT se realiza en algún momento entre las etapas 3 y 4 . La empresa receptora trabaja entonces en un prototipo preindustrial por lo que a esta etapa se le conoce como etapa precomercial en la cual también se generan las estrategias de marketing por las empresas manufactureras innovadoras. Al final de la etapa 4 inician las ventas y la difusión en el mercado del producto tecnológico con lo que se espera un flujo de recursos positivo (+\$) por las ganan- cias que se espera sean generadas por las ventas de la tecnología.

De acuerdo con la discusión anterior, las tecnologías universitarias se encuentran en algún lugar entre prototipos de concepto, de banco y /o de laboratorio. Por lo tanto, su costo no sólo debe incluir los costos de fabricación de los prototipos, sino otros costos intangibles como el tiempo del personal académico participante en los proyectos, el uso de maquinaria, bancos experimentales o equipo de pruebas especializado, los indirectos asociados al proyecto, el valor de los derechos patrimoniales y el valor estimado de las capacidades organizacionales y del capital intelectual de la organización que respalda la tecnología desarrollada.

Curiosamente, cuando se prepara una propuesta técnica económica para un proyecto, sólo se cotiza en términos de gasto corriente y de los recursos humanos o intelectuales que se espera serán requeridos para el desarrollo. Algunas veces ya se cuenta con alguna patente relacionada y este conocimiento también se evalúa y se cobra. Pero nunca se puede cobrar un proyecto en términos de las utilidades que se espera genere la tecnología o de los certificados y títulos de propiedad intelectual que resulten.

Muchas veces los proyectos tardan más tiempo del esperado o requieren la participación de más personal o materiales y equipo no considerados. Si además se consideran los costos ${ }^{4}$ de la administración local y del sistema universitario, de gestión, del aparato legal, de coordinación, de uso de la infraestructura, de vinculación, de uso del nombre universitario, entre otros, el costo final de los proyectos llega a ser cinco (5) veces más que lo cotizado; sin embargo la universidad debe mantenerse en el mercado y tener presente que el proyecto dejará otros dividendos académicos muy importantes como son la formación de recursos humanos, la generación de activos de propiedad intelectual, la generación de conocimiento y de competencias de segundo orden; es decir, aprender a aprender.

Las complejidades de la valuación tecnológica pueden verse en la metodología de la figura 2. Se requiere establecer un rango del valor con un precio base y un precio techo, posteriormente hacer un cálculo de valor intermedio para llegar a un precio final (Vega et al., 2008).

Según Contractor (2000), el valor base de la tecnología puede obtenerse a partir de la sumatoria de los diferentes costos directos de desarrollo y reproducción del

\footnotetext{
4 La fracción correspondiente al proyecto o a la fracción de tiempo utilizado para el proyecto.
} 


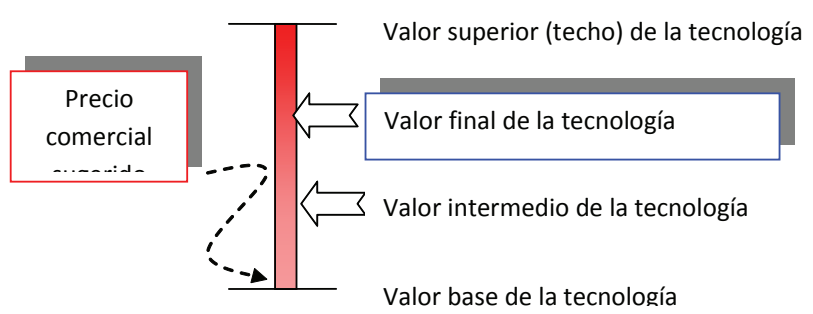

Figura 2. Metodología de valuación tecnológica

dispositivo, aunados a los costos marginales de la transferencia de conocimiento; por ejemplo, un buen costeo del desarrollo de un dispositivo de medición electrónico puede ser de unos $\$ 50,000.00 \mathrm{MN}$ más un $25 \%$ de costos de transferencia podrían llevarnos a $\$ 62,500.00$ MN. Este sería el valor base de la tecnología. Por otra parte, el valor techo de la tecnología podría determinarse a partir del cálculo de las utilidades que se esperan sobre las ventas del dispositivo a precios del mercado, dentro de su ciclo de vida. Para esto se requiere contar con estudios, o por lo menos, perfiles de mercado. Suponiendo para el ejemplo ventas totales a valor presente de $\$ 5,000,000.00 \mathrm{MN}$ durante un ciclo de vida estimado de cinco años y una utilidad de $25 \%$, para la empresa productora licenciataria de la tecnología, las normas industriales promedio señalan que la empresa debería pagar al menos un $20 \%$ de su porcentaje de utilidad a los desarrolladores; es decir, un $5 \%$ de sus utilidades totales. En el ejemplo resulta \$250,000.00 MN. Por técnicas de negociación diferentes se podría llegar al valor final de la tecnología que para el ejemplo podría ser de unos $\$ 200,000.00 \mathrm{MN}$, aproximadamente.

\section{Reflexión, comentarios y recomendaciones}

Tal vez el ánimo de los académicos cuando mencionan que su tecnología es de bajo costo sea atraer al posible cliente o patrocinador y mostrarle que la institución costea en forma justa los proyectos que desarrolla para estar dentro del mercado; sin embargo, el efecto mental en los patrocinadores es el contrario, por lo que es preferible no mencionar costos ni tratar de establecer el valor de la tecnología en una presentación corta. Lo más recomendable es dejar esto en manos de gestores tecnológicos con experiencia en valuación tecnológica. Sencillamente se puede mencionar en la presentación “...los costos de fabricación de este prototipo representan sólo un valor parcial, con mucho gusto en otra ocasión podemos definir aproximadamente el valor total de la tecnología..."

La tecnología universitaria no necesariamente debe considerarse de bajo costo. Según la teoría subjetiva del valor de la economía clásica, "una cosa no vale por lo que cuesta, sino que cuesta en virtud de su valor". (La
Gran Enciclopedia de la Economía, 2009). Más allá de su costo, el valor de una tecnología dependerá de su valor de uso. En el caso de tecnologías precompetitivas no es posible determinar con precisión su valor real, debido a que aún no se conoce el uso que hará de la misma la sociedad, por lo que el mismo debe estimarse. Obtener su valor correcto requiere además estimaciones y tanteos del uso de metodologías de valuación sustentadas en técnicas probadas; la misma debe ser realizada por personal especializado y con experiencia.

Una innovación se presenta cuando la sociedad usa una tecnología, ya que la misma se difunde, madura, adquiere un valor de cambio y por lo tanto, tiene impacto social.

En este caso también es importante que al formar investigadores los profesores concienticen a sus estudiantes sobre el valor y la dignidad que merecen sus estudios y su trabajo, así como de que deberán presentan sus trabajos y desarrollos en forma digna para evitar la impresión de que la institución es "baratera" en sus posibles patrocinadores.

La universidad también deberá formar gestores tecnológicos especializados en valuación tecnológica.

\section{Tercer mito}

El desarrollo tecnológico se logrará en unos cuantos meses.

\section{Situación alrededor del mito}

$\mathrm{Al}$ preparar una propuesta técnico económica, o bien, durante las negociaciones para la firma de un convenio para realizar un proyecto de desarrollo tecnológico patrocinado, una situación común por parte de los grupos universitarios es aceptar tiempos de entrega muy cortos para el desarrollo, presionados por los requerimientos del cliente o de las instituciones de financiamiento. Esto muchas veces se convierte en una trampa, ya que por un lado se aceptan las condiciones de entrega requeridas para no perder el proyecto, pero por el otro también el financiamiento para el proyecto generalmente es restringido. También hay que recordar que los proyectos de IA\&DT son muy diferentes a los proyectos de ingeniería, en el sentido de que las especificaciones para este tipo de proyectos generalmente son inexistentes y se limitan a una serie de ideas e intenciones por medio de las cuales el cliente explica a los desarrolladores cuál es su problema a través de generalidades sobre el dispositivo a desarrollar. Esto complica las cosas, dado que con algunas directrices preliminares los proyectos de IA\&DT son exploratorios y en ellos generalmente hay que descubrir las rutas de solución, ya que las mismas no están resueltas. Por esta razón no es 
una cuestión que se pueda resolver aumentando el número de horas hombre contratando más ingenieros para resolver el mismo problema o trabajando fines de semana y por las noches. Los proyectos de ingeniería de reversa son el mejor de los casos, ya que al existir software, dispositivos o procesos probados en alguna parte del mundo, se puede intentar su reproducción adaptándola a las necesidades locales; sin embargo, frecuentemente el desarrollo de este tipo de proyectos se vuelve muy complejo al grado de que los mismos se convierten en verdaderos dolores de cabeza para los miembros del equipo de desarrollo y para los directivos de la institución.

\section{Análisis bajo el enfoque de gestión tecnológica}

En el CCADET han existido diversas experiencias que demuestran empíricamente que los tiempos de desarrollo de proyectos innovadores requieren generalmente más de cinco años para su ejecución. Por ejemplo el proyecto "Diseño y desarrollo de equipos didácticos" solicitado por la empresa Harry Mazal SA con copatrocinio de CONACYT, en su propuesta fue previsto para su desarrollo en 2 años, el proyecto en realidad se terminó en 5 años y se convirtió en una innovación después de 7 años (Vega, 2007), Otros ejemplos similares son el probador de válvulas cardiacas desarrollado para el Instituto Nacional de Cardiología el cual se acordó para desarrollarse en dos años; sin embargo, el proyecto se extendió por dos años más. Los trabajos de puesta en marcha requirieron de otro año y los de calibración aún continúan a la fecha de este escrito. Sólo por citar un tercer proyecto, también tenemos el caso de un multigraficador de señales biomédicas desarrollado para la Facultad de Medicina de la UNAM que tomó más de dos años para su integración, aún cuando ya se contaba con desarrollos previos a la fecha de concertación del proyecto.

\section{Reflexión, comentarios y recomendaciones}

El frecuente cambio en el candelario de los proyectos de IA\&DT generalmente no se debe a indolencia o falta de seriedad. Lo que sucede es que en el camino del desarrollo de los proyectos de IA\&DT muchas veces se presentan problemas que no es posible prever al establecer las propuestas técnico económicas de los proyectos. Los problemas pueden ser financieros, por subestimación de costos, técnicos, administrativos, etcétera. Una de las tareas de la gestión tecnológica y de la administración de proyectos es prever estos problemas y plantear acciones contingentes.

Por supuesto que, en principio, hay que respetar los calendarios acordados en los convenios; sin embargo, como medida de seguridad para la institución, los tiempos que se ofrecen al cotizar proyectos de IA\&DT siempre deben ser propuestos en calidad de "estimados" negociando con la contraparte que los mismos podrán ajustarse según los hallazgos que vayan surgiendo a lo largo del avance del proyecto. También hay que solicitar que personal de la organización patrocinadora se involucre con el equipo de trabajo, a fin de que puedan mantenerse reuniones de control de avance con la mayor frecuencia posible; esto es, mensual, quincenal, semanalmente, o inclusive diariamente, dependiendo del caso. Esta práctica permitirá la toma oportuna de decisiones, lo cual evitará demoras en las entregas. Las entidades universitarias que ofrecen el desarrollo de proyectos de IA \&DT deben contar con el capital intelectual adecuado, equipos de desarrollo experimentados con capacidades y pericias adquiridas, a través de diversas experiencias en proyectos de desarrollo. Finalmente, aún para una organización que cuenta con el capital intelectual y la infraestructura adecuada, es imposible determinar a priori el tiempo total que requerirá el desarrollo de un paquete tecnológico. La estrategia de mayor seguridad es dividir el proyecto en fases y empezar por la fase de prueba de concepto, en la cual se determinará la factibilidad técnico-económica del producto tecnológico solicitado y permitirá hacer una mejor estimación de los tiempos requeridos para llegar a término.

\section{Cuarto mito}

Para el proyecto no se requiere gestor de tecnología, la gestión la hacen los jefes de laboratorio sobre la marcha.

\section{Situación alrededor del mito}

En la etapa de gestación de proyectos, una vez que se han discutido las especificaciones con el cliente y se ha llegado a la definición de un equipo de trabajo que puede hacer frente al desarrollo tecnológico solicitado, llega el momento de elaborar la propuesta técnico-económica. En esta propuesta generalmente se toma en cuenta el costo del tiempo de los académicos y del equipo técnico de apoyo que trabajará en el proyecto. En algunas instituciones se cuenta con políticas de pago de ingresos extraordinarios para motivar la participación de los académicos en este tipo de proyectos. Además se incluyen todos aquellos conceptos de gasto corriente, materiales de consumo, viáticos, equipo de cómputo y/o de laboratorio, becarios y los indirectos de las entidades. Pero casi nunca se incluyen los costos u honorarios para el equipo de administración, alegando que esto encarecería el proyecto y haría la propuesta poco 
competitiva en el mercado. En consecuencia, tampoco se consideran costos u honorarios del gestor tecnológico. Sin embargo, casi siempre los proyectos de desarrollo tecnológico por su complejidad requieren la aplicación de técnicas de control de proyectos y la aplicación de estrategias económicas y de negocios llevadas a cabo por gente experimentada en el asunto.

Peor aún, hemos tenido experiencias en las que al solicitar el financiamiento de proyectos por medio del programa de financiamiento gubernamental, como el Sectorial de Salud de CONACYT, al solicitar recursos para el pago de un gestor tecnológico para proyectos de envergadura considerable, ni el personal técnico del propio centro participante en la propuesta, ni el personal encargado del Fondo CONACYT estuvieron de acuerdo en que el pago de un gestor tecnológico fuera un rubro financiable, porque según ellos, dicho personaje no contribuiría con el desarrollo del proyecto y sólo lo encarecería.

La experiencia demuestra que casi siempre en los casos en los que los jefes de laboratorio o el personal académico pretenden realizar esta función, el resultado es que los mismos siempre se ven muy ocupados y agobiados por sus actividades académicas, por lo que con el tiempo se presentan problemas con las fechas de entrega, con los entregables incompletos y una serie de situaciones que llevan a la no satisfacción de los clientes patrocinadores. En algunos casos extremos se presentan demandas cuando los patrocinadores no reciben el producto que ellos pidieron o especificaron.

\section{Análisis bajo el enfoque de gestión tecnológica}

Las situaciones problemáticas que pueden darse durante el desarrollo y al final de los proyectos de IA\&DT tienden a minimizarse e inclusive prácticamente se anulan cuando se aplican técnicas de gestión tecnológica adecuadamente.

La razón es que el quehacer de un gerente de proyectos o gestor tecnológico tiene que ver con proyectos de largo alcance, duración y niveles de complejidad. Enfoca los proyectos no sólo desde la perspectiva técnica o del trabajo físico involucrado, sino, también como procesos de negocios, siendo estos últimos considerados en la actualidad como procesos fundamentales de la mayoría de las organizaciones (Maylor, 2001).

Si bien en México estos conocimientos y procesos de gestión no son bien comprendidos, a nivel internacional la situación es diferente. Por ejemplo, en un estudio realizado a dos proyectos de desarrollo de Ericsson Ra- dio y Volvo Car Corporation, se encontró que los papeles clave del gerente tecnológico de proyectos son:

a) La integración de conocimiento multidisciplinario,

b) el manejo sistémico del conocimiento ya que consideran al proyecto como un gran todo,

c) la administración y control de las actividades en las que se genera el conocimiento,

d) la sincronización del tiempo, ya que conducen los trabajos para mantener las fechas de entrega a tiempo,

e) el ajuste de tiempos -es un papel más orientado a la acción, ya que coordina a los participantes y los apura para cumplir con metas y fechas,

f) la generación intuitiva de ritmo para las juntas que deben llevarse a cabo entre los diversos participantes del proyecto y los clientes principales (Söderlund, 2005).

\section{Reflexión, comentarios y recomendaciones}

El desarrollo de nuevos y múltiples productos tecnológicos cada vez más requerirá de la fusión e integración del conocimiento científico y tecnológico disponible en áreas del conocimiento, tales como los nuevos materiales, la mecánica, la electrónica, la bioelectrónica, la nanotecnología y la manufactura inteligente, entre muchas otras. Esto a su vez, dará pie a nuevos productos más complicados en un ciclo interminable. El proceso requerirá que los ingenieros, el personal técnico, los académicos, los administradores y el personal de apoyo se coordinen con los gestores de tecnología para adaptarse al mundo multidisciplinario a través de procedimientos interculturales de comunicación, cooperación y el control de proyectos. La labor del gestor de tecnología debería ser imprescindible para el buen desarrollo y término de los proyectos, aunque en nuestro medio es muy poco comprendida.

Una posible solución es que en las diferentes entidades de la UNAM se cuente con gestores tecnológicos especializados que puedan llevar a cabo la administración de los proyectos de su entidad. En algunos casos se pueden establecer bases de colaboración interinstitucional con el fin de que gestores establecidos en alguna de las entidades puedan colaborar apoyando la gestión de proyectos de otras entidades que no cuenten con estas capacidades.

\section{Quinto mito}

Hay que patentar todos los desarrollos de la UNAM, porque así se hace en otras instituciones como el Instituto Tecnológico de Massachussets (MIT) y en otros países. 
Situación alrededor del mito

En los discursos oficiales pronunciados por diferentes autoridades, es muy frecuente oír comparaciones que se hacen entre el número de patentes mexicanas, particularmente las universitarias y el número de patentes que obtienen otros países e instituciones. Sin embargo, generalmente en este tipo de pronunciamientos nunca se menciona ni se explica cuáles deben ser las fuentes de financiamiento para sufragar los costos de obtención, administración, vigilancia y litigios por invasión de patentes.

Los objetivos de la Universidad Nacional Autónoma de México (UNAM) son la formación de recursos humanos, la investigación y la difusión de la cultura. También se realizan intensos esfuerzos de investigación y desarrollo en el Subsistema de Investigación, mientras que las facultades y escuelas realizan investigación relacionada con la enseñanza y con la demostración de principios básicos de la física, la química, la ingeniería, la medicina y las ciencias biológicas, entre otras. Se han obtenido muy buenos resultados en ciencia básica, (CIC-UNAM, 2008) y se ha desarrollado tecnología precompetitiva con financiamiento interno para los procesos experimentales y de enseñanza (CCADET-UNAM, 2008).

La mayor parte de la tecnología universitaria es precompetitiva y su propósito general es diferente al de la comercialización. Algunas veces los desarrollos son financiados por la industria a través de la firma de convenios de colaboración y de desarrollo tecnológico. Los resultados toman la forma de prototipos de laboratorio para los cuales, una vez que son terminados y probados se obtienen patentes, derechos de autor o marcas que incrementan los derechos patrimoniales de la UNAM. En términos generales, la tecnología universitaria se encuentra en el campo de las innovaciones incrementales, ya que está directamente relacionada con mejoras del desempeño de algunas tecnologías o con mejoras a algunas características de productos ya existentes (Hacklin et al., 2004). En muy raros casos se trata de tecnologías de ruptura.

Análisis bajo el enfoque de gestión tecnológica

El número de patentes que obtiene una organización habla más de su capacidad de inventar que de su capacidad de innovar, es decir, habla más de la capacidad que se tiene de inventar que de la actitud de los agentes de la organización para involucrarse en proyectos de innovación. Una gran proporción de patentes nunca son comercializadas porque tienen una vida de "anaquel" específica. Por ejemplo, el Centro de Distribución de Patentes (PADIC) de Japón, estima que el 65\% de más de un millón de patentes japonesas están dormidas o son redundantes sin producir valor para sus dueños. Por otra parte, el número de patentes de la empresa $3 \mathrm{M}$ se redujo un $24 \%$ en 1999 , respecto al año anterior, pero en el mismo año se registró un incremento en el número de nuevos productos que la firma introdujo al mercado. De acuerdo con la alta gerencia de la empresa, la estrategia fue la de enfocarse en las áreas de tecnologías críticas y comercializarlas más eficientemente ( $\mathrm{O}^{\prime}$ Donoghue \&Scotchmer, citados por Collinson et al., 2005).

En la administración de la propiedad intelectual se presenta un fenómeno curioso: las organizaciones a nivel mundial, generalmente obtienen un gran número de patentes de importancia marginal y un número muy pequeño de patentes de alto valor y alto impacto. Por lo tanto, se requiere un análisis muy serio de patentes para enfocar el tiempo, los recursos y los esfuerzos a aquellas patentes y actividades que agreguen mayor valor a los derechos patrimoniales de la organización. (Breitzman et al., 2002).

Por otra parte, existe la creencia de que el hecho de obtener un número masivo de patentes es sinónimo de que se realiza buena ciencia y tecnología; sin embargo, no sólo se trata del número de patentes sino también de la calidad de las mismas. Una medida de su calidad es el número de citas que reciben las patentes. El puntaje de citas (potential store) es una medida que permite decidir si se deben continuar pagando las tarifas de mantenimiento de derechos anuales. También se debe formular una estrategia de propiedad intelectual, para lo cual hay que definir dominios de propiedad importantes para la organización y después analizar el banco de patentes vigentes para responder a preguntas como las siguientes: ¿es esta patente todavía importante en nuestro dominio de propiedad intelectual?, ¿nuestro programa de IA\&DT sigue construyendo alrededor de ella?, si la patente no ha recibido otras citas o auto citas esto sugiere que la misma tiene poco valor (Breitzman et al., op. cit.).

\section{Reflexión, comentarios y recomendaciones}

Por razones de costos y de estrategia tecnológica organizacional, siempre será más razonable patentar solamente aquellos desarrollos que tengan potencial comercial, los cuales se descubrirán través del análisis de patentabilidad y del análisis de mercado respectivo. No es posible mantener una cartera demasiado extensa de patentes no productivas, ya que no existe una fuente de financiamiento específica para cubrir los costos asociados. En nuestra opinión, los escasos recursos financieros deben dirigirse a continuar con las labores de 
desarrollo, investigación y enseñanza. La organización debe generar una estrategia de propiedad intelectual congruente con sus objetivos y sus capacidades. Sólo aquellos desarrollos tecnológicos que de acuerdo a la estrategia y objetivos organizacionales tengan un alto valor comercial o estratégico deberán patentarse.

\section{Sexto mito}

La generación de conocimiento no requiere administración.

\section{Situación alrededor del mito}

La gerencia de tecnología (Technology Management), en su concepto más amplio no sólo incluye actividades y técnicas de gestión de proyectos, sino que es un proceso que incluye planeación, dirección, control y coordinación del desarrollo e implementación de las capacidades tecnológicas que tiene una organización para lograr sus objetivos operacionales, lo que ha sido reconocido desde fines del siglo pasado como una actividad crucial para la industria y el gobierno (Rubinstein, citado por Linn, et al., 2000). Hoy en día se sabe que un manejo apropiado de la tecnología es lo que crea riqueza para las naciones, las compañías y los individuos. Es el fundamento del crecimiento económico y determina la competitividad nacional y organizacional, particularmente en un mercado global intensamente competido (Khalil et al., 2005).

Por otra parte, según West et al. (2003), la investigación y el desarrollo tecnológico se basan en la acumulación de experiencias y en la experimentación, por lo que fundamentalmente son actividades de generación y retención de conocimiento. Los proyectos de IA\&DT requieren de la interacción de diferentes actores, grupos interdisciplinarios de académicos, personal técnico, administrativo y de apoyo. También necesitan procedimientos para el uso adecuado de la infraestructura, procedimientos logísticos para la adquisición y manejo de materiales y la coordinación y control de todos los recursos humanos, materiales y financieros. Por diferentes razones culturales, en nuestro medio, las actividades de IA\&DT se han visto muy alejadas de los procedimientos de administración. Incluso las áreas económico-administrativas son consideradas como de menor rango entre los científicos. En estos círculos frecuentemente se escucha que la persona que realiza estudios de posgrado en administración, planeación, gestión tecnológica de proyectos ó economía, realizó un posgrado "patito".
Análisis bajo el enfoque de gestión tecnológica

Los sistemas de IA\&DT han tenido una evolución continua a lo largo del tiempo. La primera generación se remonta desde inicios del siglo pasado hasta los años sesenta. Sus características principales eran que se basaban fundamentalmente en la ciencia y ponían muy poca atención a la administración económica. La segunda generación se enmarcó entre los años 1970 y 1980's y fue caracterizada por su enfoque muy científico y administración estratégica, mientras que la tercera generación fue vigente entre 1990 hasta el año 2000 en la que se introdujo una administración estratégica y holística. La cuarta generación de sistemas de IA\&DT emergió con este siglo, (Millar \& Morris citados por Park et al., 2006). El objetivo principal de la cuarta generación de sistemas de IA\&DT fue identificar las necesidades latentes de los clientes potenciales y asegurar simultáneamente la factibilidad técnica y comercial en las etapas tempranas de los proyectos. Según estos autores, el proceso de IA\&DT puede ser considerado primariamente como un proceso de administración del conocimiento porque transforma la información en avances tecnológicos y las demandas del mercado en conocimientos que pueden ser usados para desarrollar nuevos procesos, diseños y productos.

\section{Reflexión, comentarios y recomendaciones}

Siendo las actividades de investigación y desarrollo tecnológico, generadoras fundamentales del conocimiento en el contexto de una organización, requieren de la aplicación de la gerencia tecnológica, ya que ésta última integra las diferentes estrategias tecnológicas y de negocios de la misma, (Khalil, op. Cit.). Instituciones de fomento como el Consejo Nacional de Ciencia y Tecnología (CONACYT) y el Instituto de Ciencia y Tecnología del Gobierno del Distrito Federal (ICYTDF) deberían considerar la necesidad de promover la formación de gestores tecnológicos y administradores de proyectos en diferentes especialidades, a través de la promoción y otorgamiento de becas de estudios.

\section{Séptimo mito}

Es muy fácil crear riqueza a partir de la comercialización de los resultados de la investigación.

\section{Situación alrededor del mito}

En México, como en todas partes del mundo, hoy en día estamos viviendo la emergencia continua de nuevos productos tecnológicos a una tasa acelerada, impulsada por fuerzas dinámicas como la aceleración del 
cambio tecnológico, las demandas crecientes de los clientes, las restricciones de los sistemas de calidad total, la competencia global, la reingeniería, los sistemas de información compartida y las regulaciones gubernamentales (Hughes et al., 1996).

Como se mencionó previamente, el desarrollo de nuevas tecnologías en las universidades puede tener dos tipos de objetivos: a) académicos y b) el desarrollo de nuevos productos (NPD, por sus siglas en inglés) con un destino comercial. Hasta hoy en México, el proceso de desarrollo, de nuevos productos en empresas, así como en Centros e Institutos de Investigación y Desarrollo, tanto públicos como privados, ha consistido de una serie de etapas discretas generalmente secuenciales, mientras que el desarrollo de nuevos productos tecnológicos requiere ser un proceso continuo de etapas concurrentes, dado que poco a poco nos movemos más hacia una era de innovación continua (Ángel, 1994).

Según Marinova y Phillimore (2003), a fines de la última década del siglo pasado, existían al menos seis tipos diferentes de modelos de innovación a nivel global, que son los siguientes:

a) Primera generación de la caja Negra (1960-1970),

b) segunda generación, modelos lineales del empuje tecnológico y jalón de la demanda (1970-1980),

c) tercera generación de modelos interactivos de acoplamiento e integración (1985),

d) cuarta generación de modelos sistémicos de redes y sistemas nacionales de innovación (1990-1995),

e) quinta generación de modelos evolucionarios (19952000),

f) sexta generación de modelos de mileaux innovativo (2000-2005).

Sólo hablamos de innovación tecnológica cuando la tecnología alcanza el mercado, es aceptada y pagada por los clientes y por lo tanto, se crea riqueza (Khalil et al., op. cit.).

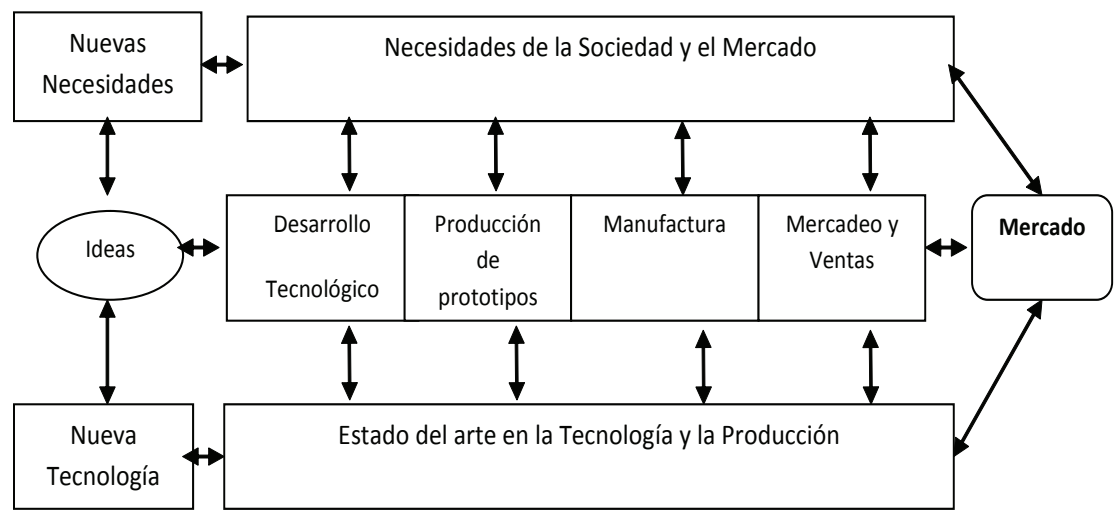

Análisis bajo el enfoque de gestión tecnológica

En la figura 3, se muestra el modelo de innovación tecnológica clásico de los años 90 del siglo pasado (Rothwell, 1991). En este modelo puede verse que el origen del proceso son las ideas alimentadas por las demandas del mercado y por la emergencia de nuevas tecnologías y que el fin último del proceso es el mercado. También podemos ver que en el corazón del proceso de innovación tecnológica se encuentra el proceso de desarrollo tecnológico de prototipos funcionales que después dan origen a la producción de prototipos y a procesos de manufactura y de mercadeo.

El proceso en su conjunto es extraordinariamente complejo. Baste notar que las organizaciones que fijan dentro de sus objetivos y metas desarrollar tecnología requieren entre otras cosas:

a) Reducir el tiempo de entrega de los prototipos preindustriales,

b) reducir el tiempo para el desarrollo de nuevos productos,

c) disminuir los costos de desarrollo manteniendo el alto nivel de calidad del producto,

d) entregar siempre los productos adecuados satisfaciendo los requerimientos cambiantes de los clientes,

e) incrementar la agilidad de la organización para introducir procesos innovadores como respuesta a la evolución requerida por el medio ambiente (mercado, reglamentaciones, estrategias, evoluciones tecnológicas),

f) establecer mecanismos para la comercialización de los resultados de la investigación,

g) incrementar la innovación en el nivel del servicio al cliente,

h) participar en los sistemas de innovación, aunque estos sean incipientes (Segarra, 1999).

El proceso de desarrollo tecnológico también requiere aprendizaje organizacional haciendo énfasis en una visión gerencial, liderazgo, comunicación y de trabajo en equipo (Sengue, 1990). Para mejorar la efectividad de la organización a través de los procesos de aprendizaje, también se requiere de establecer una memoria organizacional que permita guiar las acciones de la misma (Robey et al., 2000).

Figura 3. Modelo de innovación de 3ạ . Generación lineal 
En México son muy pocas las instituciones públicas, privadas y las empresas que desarrollan tecnología. Los sistemas de apoyo y financiamiento gubernamentales son escasos por lo que según Dutrenit (1994), aún no se ha llegado a la definición de un Sistema Nacional de Innovación, en el sentido de la introducción comercial de progreso técnico.

En un estudio del Banco Mundial, se afirma que el Sistema Nacional de Innovación (SIN) en México, ha sido ineficiente en la acción de transformar las inversiones en IA\&DT en ideas comerciales y económicamente significativas. También se indica que el desempeño de México ha sido deficiente en innovación, debido en parte a sus bajas inversiones en IA\&DT y a la falta de cooperación entre la empresa privada y los investigadores en las universidades (Lederman, 2006).

A nivel organizacional, generalmente se han usado siete medidas de desempeño que son: efectividad, eficiencia, calidad, productividad, calidad de la vida de trabajo, posibilidad de obtención de utilidades (profitability) e innovación (Kumpe \& Bolwijn, citados por Choi et al., 2005).

Por otra parte, dado que el desempeño puede ser definido como un concepto que toma diferentes significados en diferentes situaciones para diferentes sistemas organizacionales, se pueden considerar como un mínimo de indicadores de desempeño, la eficiencia, la calidad, el tiempo de los procesos, la innovación y la contribución a la obtención de utilidades (Kaplan \& Norton, citados por Choi et al. op. cit.).

La ingeniería industrial, la económica y la administración han propuesto diversas modalidades para la medición de los indicadores de eficiencia, calidad, tiempo de procesos y contribución a las utilidades, mientras que en un sentido más amplio, la medida fundamental del éxito en la innovación es la tasa de crecimiento del ingreso per cápita y por lo tanto, del bienestar de la población.

\section{Reflexión, comentarios y recomendaciones}

Para incrementar el número de instituciones públicas y privadas así como empresas que desarrollen tecnología en México, es necesario, y por lo tanto muy recomendable, incrementar los apoyos financieros a través de fondos de fomento que funcionen de manera ágil y sin trabas burocráticas. Al disponer de recursos financieros se habrá vencido uno de los principales obstáculos. Enseguida será necesario trabajar en diversos aspectos de la cultura social, empresarial y organizacional. Por ejemplo, no obstante que aprender a desarrollar tecnología es parte fundamental del proceso de innovación tecnológica, en México las empresas y las universida- des aún no han aprendido a trabajar en conjunto para desarrollar tecnología con objetivos comerciales. Por lo tanto, estrechar las relaciones y la comunicación entre los universitarios que desarrollan tecnología con los empresarios y el mercado es necesario para propiciar la innovación tecnológica. De acuerdo con Hindle et al. (2004), estos mecanismos de comunicación e interacción son vitales para la creación y el mantenimiento de la riqueza de cada nación.

Los productos de la innovación se miden internacionalmente con el índice IA\&DT/PIB, donde IA\&DT es el porcentaje del PIB nacional aplicado en investigación y desarrollo y PIB es el total del Producto Interno Bruto. En el caso de México esta razón o cociente es uno de los más bajos en el ámbito mundial. Lederman (op.cit.), no parece aventurado al afirmar que el resultado sería todavía menor si calculáramos el número de innovaciones o productos desarrollados que alcanzaron el mercado entre el total del PIB.

Ante los hechos, parece quedar claro que en México sólo algunas grandes empresas apenas han incursionado en modelos integrados interactivos de innovación de tercera generación, mientras que en la mayoría de micro y pequeñas empresas siguen navegando en modelos de innovación de segunda generación, es decir, impulsados por el empuje tecnológico o la demanda del mercado. Estamos muy lejos de llegar a un Sistema Nacional de Innovación, el cual es característico de los modelos de innovación de cuarta generación, muy de moda en los países desarrollados. Hay casos de innovación muy renombrados y reconocidos como son el acero esponja de HYLSA de los años 80, la píldora anticonceptiva de Sintex de los años 90, el suero antialacrán de PROBIOMED comercializado por Laboratorios Silanes desde el último cuarto del siglo pasado y la tecnología de anticoagulantes sanguíneos transferida a Shering Plough por el Instituto de Biotecnología de la UNAM, por citar algunos ejemplos; sin embargo, en la mayoría de los casos, los proyectos de innovación han tenido resultados discretos muy locales con impacto social muy restringido. Comercializar desarrollos tecnológicos y generar riqueza o alto impacto social no es de ninguna manera fácil.

\section{Conclusiones y recomendaciones}

Según Khalil et al. (op. cit), en los países en desarrollo hay que tomar en cuenta dos aspectos fundamentales, el primero es que no importa si se trata de alta tecnología o de baja tecnología, lo que importa es que sea la tecnología correcta; y el segundo es que el juego global va a ser ganado en términos de velocidad y de agilidad. 
Si pudiéramos generalizar las situaciones a través de las cuales se presentaron y analizaron los mitos, podríamos inferir que si en las universidades públicas mexicanas y tal vez inclusive en las Latinoamericanas, aprender a desarrollar tecnología es difícil, lo será aún más enfocarse a la tecnología correcta y comercializarla.

Para que la universidad cumpla con su misión contribuyendo en la solución de los grandes problemas nacionales, las entidades universitarias deben aprender a desarrollar tecnología, asimilarla, adaptarla y utilizarla para obtener su mayor beneficio e impacto social. Para esto, se requiere que los directivos así como los consejos académicos y técnicos de las diferentes entidades de la UNAM, cambien sus formas tradicionales de evaluar, en las que fundamentalmente se consideran como elementos para el puntaje que se usa para los procedimientos de contratación, promoción y estímulos, la publicación de resultados de investigación y los aspectos relativos a la formación de recursos humanos.

Es necesario respetar la pluralidad en la UNAM, en el sentido descrito por Sefchovich, (2009), una manera de hacerlo es valorando adecuadamente la participación de los académicos en los proyectos de IA\&DT. Las comisiones dictaminadoras deberán considerar la importancia y la trascendencia del trabajo que en particular desarrollen los académicos en dichos proyectos, considerando aspectos tales como la complejidad del mismo, los tiempos requeridos y las diversas problemáticas organizacionales y financieras involucradas. La valoración deberá realizarse teniendo como marco de referencia los impactos sociales y económicos esperados a partir de los resultados de los proyectos. Con todo esto se promoverá una actitud de innovatividad positiva entre los académicos dirigida a fomentar el desarrollo de proyectos de IA\&DT y se estrecharán las relaciones y la comunicación entre los universitarios que desarrollan tecnología con los empresarios, el mercado y los representantes de las organizaciones productivas.

Las tecnologías universitarias deben dejar de tener solamente valor de uso como dispositivos académicos a través de los cuales se forman y gradúan licenciados, maestros y doctores. Para que las mismas tengan un mayor significado para la sociedad también deberán de tener valor de cambio y contribuir en la solución de problemas específicos y en la promoción de la economía nacional, por lo tanto, deben ser comercializables; sin embargo, comercializar desarrollos tecnológicos de alto impacto social y generar riqueza no es una tarea fácil.

Dado que la gerencia tecnológica integra las diferentes estrategias tecnológicas y de negocios, resulta evidente la necesidad de aplicar técnicas de gestión tecnológica para la administración de proyectos para asegurar la ade- cuada protección del patrimonio intelectual y para realizar la adecuada valuación de los resultados tecnológicos.

No debemos pensar en la tecnología universitaria como tecnología barata o de bajo costo. El valor real de la tecnología universitaria va más allá de sus costos de desarrollo ya que, como hemos dicho, su objetivo puede ser comercial o académico. Para determinar su valor real se requiere de la aplicación de metodologías de valuación sustentadas en técnicas probadas; las mismas deben de ser realizadas por personal especializado y con experiencia.

La labor del gestor de tecnología debería ser imprescindible para el buen desarrollo y término de los proyectos. Lamentablemente en el medio universitario y en las empresas mexicanas es muy poco comprendida y valorada.

\section{Referencias}

Ángel D.P. Restructuring for Innovation. New York. Guilford Publications. 1994.

Breitzman A.F., Mogee M.E. The Many Applications of Patent Analysis. Journal of Information Science, 28(3):187-205. 2002.

Centro de Ciencias Aplicadas y Desarrollo Tecnológico[en línea]. [fecha de consulta 10 julio de 2008]. Disponible en: http//:www. ccadet.unam.mx,).

Centro de Tecnología Avanzada CIATEQ [en línea]. [fecha de consulta 8 septiembre 2008]. Disponible en: http://www.conacyt.mx/Centros/Anuarios/2003/CIATEQ_2003.pdf.

Centro de Investigación y Asistencia en Tecnología y Diseño del Estado de Jalisco [en línea]. [fecha de consulta 8 septiembre 2008]. Disponible en: http://www.ciatej.net.mx/ ciatej.aspx

Choi D.O., Kim J.S. Productivity Measurement and Evaluation Models with Application to a Military R\&D Organization. International Journal of Technology Management, 32(3,4):408-436. Inderscience Enterprises Ltd. 2005.

Contractor F.J. Valuing Corporate Knowledge and Intangible Assets: Some General Principles. Knowledge \& Process Management, 7(4):242-255. 2000.

Coordinación de la Investigación Científica. La Ciencia en la UNAM. 1ª Edición. 2008.

Collison S., Kato H., Yoshihara H. Technology Strategy Revealed: Patterns Influences of Patent-licensing Behaviour in Japanese Firms. International Journal of Technology Management, 30(3,4):327-350. Inderscience Enterprises Ltd. 2005.

Cruells J., Difiorri A., Sistemas Productivos [en línea]. [fecha de consulta 2 septiembre 2008]. Disponible en: http://rinconcreativo.com.ar/sistemas/Tema\%2018_05.htm

Dutrenit G. Sistemas nacionales de innovación, espacios para la competitividad [en línea]. Comercio Exterior, 44(8), agosto 1994, en Carlota Pérez. [fecha de consulta 2006]. Disponible 
en: http://www.carlotaperez.org/Articulos/4-sistemanacional. htm

Goldsmith R.E., Foxall R.G. The Measurement of Innovativeness. The International Handbook on Innovation. Edited by Larisa V. Shavinina. Elsevier Science Ltd. 2003. Pp.321-330.

Grupp H., Linstone H.A. National Technology Foresight Activities Around the Globe: Resurrection and New Paradigms. Technology Forecasting and Social Change. 60:85-94. Elsevier Science Inc. North Holland. 1998.

Hacklin F., Raurich V., Marxt C. How Incremental Innovation becomes Disruptive: The Case of Technology Convergence. International Engineering Management Conference, IEEE, pp. 32-36. 2004.

Hindle K., Yencken J. Public Research Commercialization, Entrepreneurship and New Technology Based Firms: an Integrated Model. Technovation, 24:793-803. 2004.

Hughes D., Chafin D. Turning New Product Development into a Continuous Learning Process. Journal of Product Innovation Management, 13:89-104. Elsevier Science Inc. 1996.

Khalil T.M., Ezzat H.A. Management of Thecnology and Responsive Policies in a New Eeconomy. International Journal of Technology Management, 32(1,2):88-111. Inderscience Enterprises Ltd. 2005.

La Gran Enciclopedia de la Economía 2009. Valor de uso y valor de cambio [en línea]. [fecha de consulta 2 de enero 2009]. Disponible en: http://www.economia48.com/spa/d/valor-de-usoy-valor-de-cambio/valor-de-uso-y-valor-de-cambio.htm

Lederman D. Innovación en México, World Bank, Oficina del Economista en Jefe para América Latina y el Caribe [en línea]. [fecha de consulta junio de 2006]. Disponible en: http:// wbln0018.worldbank.org/lac/lacinfoclient.nsf/d296849511749 75c85256735007fef12/f7cde0329889f57d85256dc50056268a/\$FI LE/Mexico_Innovation\%20Brief_spa.pdf

Linn R.J., Zhang W., Li Z. An Intelligent Management System for Technology Management. Computers \& Industrial Engineering, 38:397-412. Elsevier Science Ltd. 2000.

Marinova D., Phillimore J. Models of Innovation. In: The International Handbook of Innovation. Edited by Larisa V. Shavinina, pp. 44-53, Elsevier Science Ltd. 2003.
Maylor H. Beyond the Gantt Chart: Project Management Moving. European Management Journal, 19(1):92-100. Elsevier Science Ltd. 2001.

Park Y., Kim S. Knowledge Management System for Fourth Generation R\&D: KNOWVATION, Technovation, Vol. 26; pp. 595602. Elsevier Ltd. 2006.

Premio Eureka, Universidad de los Andes, Venezuela [en línea]. [fecha de consulta 8 septiembre 2008]. Disponible en: http:// viceacademico.ula.ve/modules.php?op=modload\&name=Ne ws\&file $=$ article $\&$ sid $=127$

Robey D., Boudreau M.C., Rose G.M. Information Technology and Organizational Learning: a Review and Assessment of Research. Accounting Management And Information Technologies, 10:125-155. 2000.

Rothwell R. Successful Industrial Innovation: Critical Factors for the 1990's. R\&D Management, 22(3):221-239. 1991.

Scandizzo P.L. Financing Technology: an Assessment of Theory and Practice. International Journal of Technology Management, 32(1,2):1-33. Inderscience Enterprises Ltd. 2005.

Sefchovich S. Carta al Rector de la UNAM. El Universal, 11 de enero de 2009.

Segarra G. The Advanced Information Technology Innovation Roadmap. Computers in Industry, 40:185-195. Elsevier Science B.V.1999.

Sengue P. The Fifth Discipline: the Art and Practice of the Learning Organization. New York. Dobleday. 1990.

Söderlund J. What Project Management Really is About: Alternative Perspectives on the Role and Practice of Project Management. International Journal of Technology Management, 32(3,4): 371-387. Inderscience Enterprises Ltd. 2005.

Vega-González L.R., Saniger B.J.M., Qureshi N. Methodology for Pre-competitive Technology Valuation. Mimeo. 2008.

Vega-González L.R. Navegando entre rápidos, la gestión del conocimiento en proyectos de innovación tecnológica. El caso del proyecto de "Diseño y desarrollo de equipos didácticos. XI Seminario Latino-Americano de Gestión Tecnológica ALTEC 2007, 26 al 28 de septiembre, Buenos Aires, Argentina.

West J., Iansati M. Experience, Experimentation and the Accumulation of Knowledge: the Evolution of R\&D in the Semiconductor Industry. Research Policy, 32:809-825. Elsevier Science. 2003.

\section{Semblanza del autor}

Luís Roberto Vega-González. Es ingeniero mecánico electricista por la Facultad de Ingeniería de la Universidad Nacional Autónoma de México (UNAM). Obtuvo la maestría en ingeniería de sistemas en el área de planeación por la División de Estudios de Posgrado de la Facultad de Ingeniería, UNAM y la maestría en administración de las organizaciones por la División de Estudios de Posgrado de la Facultad de Contaduría y Administración de la UNAM. Se especializó en gestión tecnológica por el Centro de Innovación Tecnológica y la Facultad de Contaduría y Administración de la UNAM. Ha colaborado en firmas nacionales e internacionales en proyectos de ingeniería e integración tecnológica. Desde 1993, es académico de tiempo completo de la UNAM donde ha colaborado en el Centro para la Innovación Tecnológica, el Instituto de Ingeniería y el Centro de Instrumentos. Es coordinador de vinculación y gestión tecnológica del Centro de Ciencias Aplicadas y Desarrollo Tecnológico (CCADET). Ha conducido negociaciones, convenios, contratos y realizado actividades de administración tecnológica para cerca de dos centenares de proyectos en la UNAM. Es autor de más de un centenar de productos académicos en sus diferentes modalidades. 